\title{
Inter- and Intra-molecular Interactions of Polymers as Studied by Fluorescence Spectroscopy. V. The Effects of Degree of Oligomerization
}

\author{
Yujiro Matsuyama and Shigeo Tazuke* \\ Research Laboratory of Resources Utilization, Tokyo Institute of Technology, \\ Ookayama, Meguro-ku, Tokyo 152, Japan.
}

(Received March 5, 1976)

\begin{abstract}
KEY WORDS Fluorescence / Oligoester / Carbazole / Terephthalate / Inter- and Intra-molecular Interaction / Exciplex /
\end{abstract}

From our recent findings, ${ }^{1}$ we consider that the association of polymer chains should be one important factor in determining the fluorescence characteristics of polymer solutions. The molecular association in dilute solution by means of zipping may be closely related to the degree of polymerization; thus we prepared polyesters having a terephthalate main chain and a pendant $\omega$-(9-carbazolyl)butyl group with varying degrees of polycondensation. As anticipated, the fluorescence spectra of these polymers are molecular weight dependent.

The procedures of monomer synthesis and oligomerization have already been reported ${ }^{2}$. Monomeric model compound 2, prepared by the reaction of monomer diol with a large excess of terephthaloyl chloride, was purified by passing through a silica gel column and identified by means of IR and NMR spectroscopy: IR (neat) $1720,1270,1120,1105,1020 \mathrm{~cm}^{-1}$; NMR (acetone- $\left.d_{6}\right) \delta 1.14\left(\mathrm{~s}, 3, \mathrm{CH}_{3}\right), 1.40(\mathrm{t}, J=7 \mathrm{~Hz}, 6$, $\left.\mathrm{OCH}_{2} \mathrm{CH}_{3} \times 2\right), 1.58-1.93\left(\mathrm{~m}, 6, \mathrm{CH}_{2} \times 3\right), 4.19-$ $4.60\left(\mathrm{~m}, 10, \mathrm{CH}_{2} \mathrm{O} \times 4, \mathrm{~N}-\mathrm{CH}\right), 7.04-8.20(\mathrm{~m}$, 16 , aromatic $\mathrm{H})$. The molecular weight of the oligoesters was estimated by means of gel-permeation chromatography, using tetrahydrofuran as an eluent. Comparing the count numbers for those oligomers with that for $\mathbf{2}$, the degree of oligomerization was determined. The structure and properties of these carbazole derivatives are shown in Figure 1.

Fluorescence spectroscopy of these carbazole derivatives was conducted in dioxane at $20.0 \pm$ $0.5^{\circ} \mathrm{C}$ (excitation at $345 \mathrm{~nm}$ ). Although the

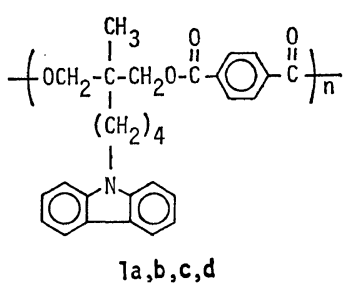

\begin{tabular}{cc} 
& $n$ \\
\hline la & 10 \\
Id & $3-5$ \\
lc & $2-4$ \\
lb & $c a .2$ \\
\hline
\end{tabular}

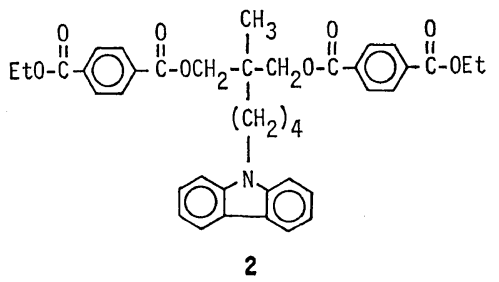

Figure 1. The structure and the degree of oligomerization of carbazole derivatives.

shapes of the emission spectra of oligomers 1a, $\mathbf{b}, \mathbf{c}, \mathbf{d}$ (Figure 2) are all similar, the intensity of the broad band around $480 \mathrm{~nm}\left(F_{\mathrm{e}}\right)$ relative to that at $368 \mathrm{~nm}\left(F_{\mathrm{m}}\right)$ is clearly different from system to system, reflecting the difference in the degree of oligomerization. This broad band is identified as the exciplex emission from the excited carbazolyl group-ground state terephthalate group pair. ${ }^{3}$ The dependence of the $F_{\mathrm{e}} / F_{\mathrm{m}}$ value on the degree of oligomerization may suggest the existence of an interaction between nonadjacent chromophores.

The fluorescence spectrum of monomeric model compound $\mathbf{2}$ is slightly different from the others. 


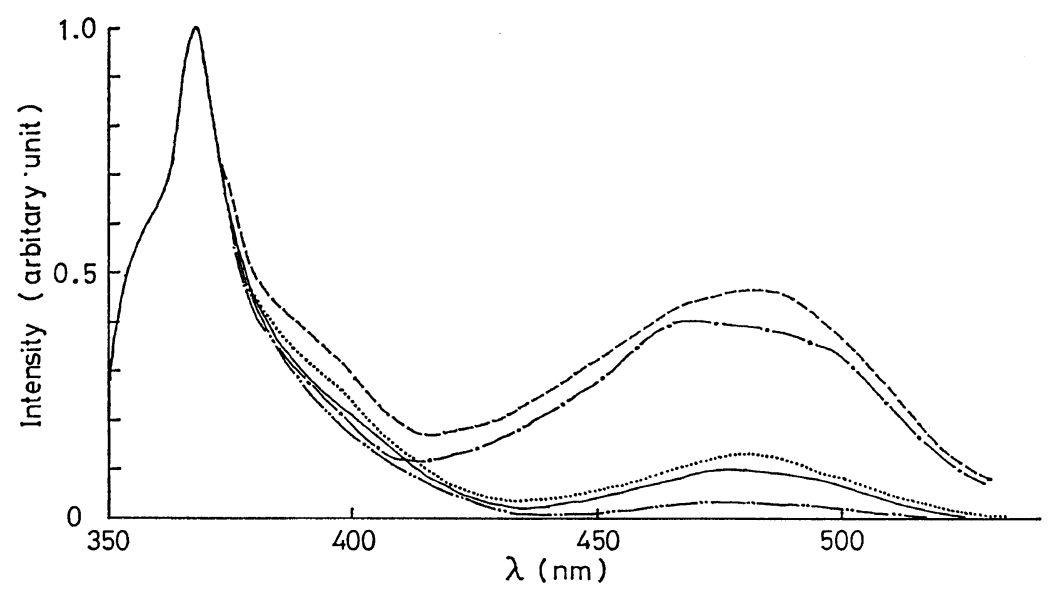

Figure 2. Fluorescence spectra of carbazole derivatives: [Fluorescer] $=1 \times 10^{-3} \mathrm{~mol} \cdot \mathrm{dm}^{-3}$; solvent, dioxane; temperature, $20.0 \pm 0.5^{\circ} \mathrm{C} ;---, \mathbf{1 a} ; \cdots, \mathbf{1 b} ;-, \mathbf{1 c} ;-\cdots-, \mathbf{1 d} ;-\cdot-, 2$.

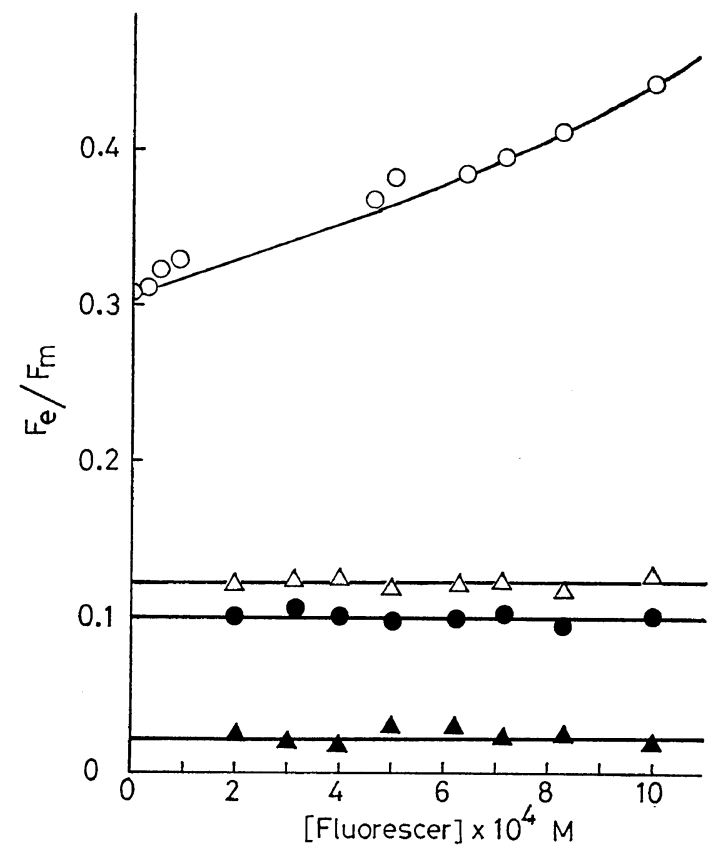

Figure 3. Concentration effect on the value of $F_{\mathrm{e}} / F_{\mathrm{m}}$ : solvent, dioxane; temperature, $20.0 \pm 0.5^{\circ} \mathrm{C}$; $-\bigcirc-, 1 \mathrm{a} ;-\triangle-, \mathbf{1 b} ;-\bigcirc-, \mathbf{1 c} ;-\Delta-, \mathbf{1 d}$.

The high exciplex intensity of 2 is an unexpected observation. Furthermore, the shape of the exciplex emission which peaks at $465 \mathrm{~nm}$ is distored and different from that of the oligomer systems, while the exciplex emission by the oligomers is identical in shape to that from the $N$-ethylcarbazole-dimethyl terephthalate pair. The difference in the shape of the exciplex emission indicates that the structure of the exciplex by 2 is different from that by the oligomers. One possibility is the formation of a sandwich-like exciplex by 2 with a carbazolyl group located between two terephthalate groups.

Marked effects of the degree of oligomerization on $F_{\mathrm{e}} / F_{\mathrm{m}}$ are observed for 1 . In addition to the highest value of $F_{\mathrm{e}} / F_{\mathrm{m}}$ observed for $\mathbf{1 a}$ at infinite dilution, the $F_{\mathrm{e}} / F_{\mathrm{m}}$ value is strongly dependent on the concentration of $\mathbf{1}$, as shown in Figure 3. The dependence of $F_{\mathrm{e}} / F_{\mathrm{m}}$ on concentration is an indication of some intermolecular association of the polymers prior to photoexcitation, as shown also by the excimer (or aggregate) formation of a naphthyl or anthryl group attached to the polyesters. ${ }^{4,5}$ The distinct difference in fluorescence behaviors of $\mathbf{1 a}$ from its lower molecular weight analogs indicates that there is a critical number of repeating units for specific interpolymer association. We consider the present results as circumstantial evidence for zipper effects in synthetic polymers, since "zipping" should become more efficient as the number of repeating units is increased.

\section{REFERENCES}

1. S. Tazuke and Y. Matsuyama, Macromolecules, 8, 280 (1975).

2. Y. Matsuyama and S. Tazuke, Makromol. Chem., 176, 1657 (1975).

3. Y. Nishijima, M. Yamamoto, and M. Ohoka, Repts. Progr. Polym. Phys. Japan, 15, 411 (1972).

4. S. Tazuke and F. Banba, Macromolecules, 9, in press.

5. S. Tazuke and F. Banba, J. Polym. Sci., Polym. Chem. Ed., 14, in press. 